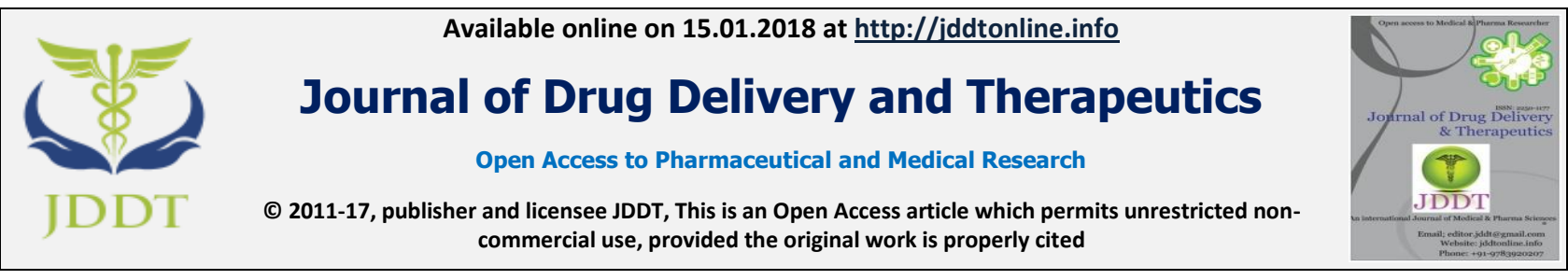

Open 6 Access

Research Article

\title{
DISSOLUTION METHOD DEVELOPMENT WITH CHROMATOGRAPHIC METHOD FOR DETERMINATION OF DRUG RELEASE IN DISSOLUTION SAMPLES OF URSODEOXYCHOLIC ACID TABLETS.
}

\author{
Tukaram B Sawant ${ }^{1,2^{*}}$, Dhananjay V Mane ${ }^{1}$ \\ ${ }^{1}$ Chhatrapati Shivaji College, Omerga, Maharashtra, India \\ ${ }^{2}$ Dr. Babasaheb Ambedkar Marathwada University, Aurangabad, 431004, MS, India
}

\begin{abstract}
Ursodeoxycholic Acid is used in the treatment of Liver disease. An accurate and robust invitro method is developed and validated for measurement of drug release in Ursodeoxycholic Acid tablets. HPLC method for quantification of drug in dissolution samples of Ursodeoxycholic Acid tablet is developed and validated. Phosphate buffer $(0.05 \mathrm{M})$ of $\mathrm{pH}-7.5$ is used as dissolution medium and paddle (USP-II) as apparatus at $75 \mathrm{rpm}$. The sample was withdrawn after 60 minutes. The developed HPLC method was used for quantitative estimation of drug release in dissolution samples of Ursodeoxycholic Acid tablet. The HPLC column of Hypersil - C18, $250 \mathrm{X} 4.6 \mathrm{~mm}, 5 \mu \mathrm{m}$ is used. The phosphate buffer of $\mathrm{pH}-6.5$ and acetonitrile was mixed in the ratio of 50:50v/v pumped at a flow rate of $1.0 \mathrm{ml} /$ minute. The detection was made at wavelength of $210 \mathrm{~nm}$. Through method validation it is proved that the method is accurate, rugged, precise and robust. The method is linear over the concentration range of $50 \%-150 \%$. The method also found very precise. Mean recovery of Ursodeoxycholic Acid was found $99.6 \pm 38 \%$.
\end{abstract}

Keywords: Ursodeoxycholic Acid, Dissolution, chromatography, Method Validation.

Article Info: Received 11 Nov, 2017; Review Completed 22 Dec, 2017; Accepted 23 Dec, 2017; Available online 15 Jan, 2018

Cite this article as:

Sawant TB, Mane DV, Dissolution method development with chromatographic method for determination of drug release in dissolution samples of ursodeoxycholic acid tablets, Journal of Drug Delivery and Therapeutics. 2018; 8(1):23-28

DOI: http://dx.doi.org/10.22270/iddt.v8i1.1547

*Address for Correspondence

Tukaram B Sawant, Chhatrapati Shivaji College, Omerga, Maharashtra, India. Email: tbsawant_916@rediffmail.com

\section{INTRODUCTION}

Ursodeoxycholic acid (UDCA), is a bile acid (fig. 1) and is generally used for the treatment of liver diseases. UDCA is a naturally occurring bile acid and used to dissolve gallstones in the gall bladder ${ }^{1-4}$.<smiles>C[C@H](CCC(=O)O)[C@H]1CC[C@H]2[C@H]3[C@H](CC[C@@]21C)[C@@]1(C)CC[C@H](O)C[C@@H]1C[C@H]3O</smiles>

Figure 1: Structure of Ursodeoxycholic Acid
It is very important to measure the drug release of UDCA for better quality of products so that the disease can be cured very fast. Hence this research was focused to develop dissolution method and measurement of drug release accurately in dissolution samples. The measurement method is chromatographic method by HPLC. There are few methods published currently for the estimation of UDCA in Ursodeoxycholic acid tablets $^{5-9}$. This method can be used in the quality control or in research laboratories of pharmaceutical companies for assay determination of Ursodeoxycholic acid tablets. The dissolution method along with chromatographic method which is used for determination of drug is validated as per International Conference on Harmonization (ICH) Q2 (R1) and United State of Pharmacopoeia (USP) chapter <1225> and chapter 
$<1092>^{10-12}$. The method found to be precise, accurate, linear and robust.

\section{EXPERIMENTAL}

\section{Materials}

Potassium hydrogen orthophosphate, Sodium hydroxide, Acetonitrile and HPLC grade water.

\section{Method and Instruments}

Dissolution apparatus of Lab India, Waters High Performance Liquid Chromatography (HPLC) system with UV detector.

\section{Dissolution method:}

Dissolution Medium: 0.05M Phosphate buffer -pH-7.5 (900 ml)

Apparatus: Paddle (USP II)

Rotation per minute (RPM): 75 RPM

Time: 60 minutes

\section{Chromatographic Parameters}

HPLC column: Hypersil - C18, 250 X 4.6 mm, 5um

Pump Flow: $1.0 \mathrm{ml} / \mathrm{min}$

Injection volume: $50.0 \mu \mathrm{L}$

Mobile Phase: Mixture of buffer and Acetonitrile in the ratio of $50: 50 \mathrm{v} / \mathrm{v}$.

Wavelength: UV detector at $210 \mathrm{~nm}$.

The column oven temperature: Ambient.

\section{Preparation of solutions:}

\section{Buffer Solution for mobile phase:}

Dissolved about $6.8 \mathrm{gm}$ of potassium hydrogen orthophosphate in $1000 \mathrm{ml}$ of HPLC grade water and adjusted the $\mathrm{pH}$ to 6.5 with dilute sodium hydroxide. Sonicated for degassing and filtered through $0.45 \mu \mathrm{m}$ membrane filter.

\section{Standard Preparation:}

Weighed accurately about 40mg of UDCA working standard and transferred to $100 \mathrm{ml}$ volumetric flask. The UDCA was dissolved in $5 \mathrm{ml}$ methanol with sonication and intermittent shaking and diluted to volume with dissolution medium.

\section{Dissolution Medium:}

Dissolved $68 \mathrm{gm}$ of potassium dihydrogen orthophosphate in $10000 \mathrm{ml}$ of purified water, adjusted $\mathrm{pH}$ to 7.5 with dilute sodium hydroxide solution. Degassed the solution with means of sonication.

\section{Sample Preparation:}

Placed one tablet in each individual jar (six tablets in six individual jars) which was contained $900 \mathrm{ml}$ of dissolution medium maintained at 37.0. The paddle was rotated at speed of $75 \mathrm{rpm}$. Aliquot was withdrawn after 60 minutes. Filtered through $0.45 \mu$ filter and injected in chromatographic system.

\section{Procedure:}

Injected blank (dissolution medium), standard solution and sample solution in HPLC system using the newly developed chromatographic method. The concentration of drug release was calculated using sample area against standard area.

\section{Method Validation}

Developed analytical method is validated for parameters as suggested by ICH Q2 (R1) and USP.

\section{System Suitability:}

System suitability test (SST) is very important in liquid chromatographic method to prove that the system is working accurately and all the chromatographic conditions are fine. They are used to verify the reproducibility of the chromatographic parameters and system is satisfactory for the analysis to be done. The system suitability test was performed in accordance with USP.

\section{Specificity:}

Specificity is the ability of the method to measure the analyte response in the presence of other substances or expected to be present. The specifity of the developed LC method was carried out by injecting dissolution medium and placebo.

The diluent (blank), placebo solution was injected in sequence for evaluation of specificity of proposed method. The chromatograms were monitored for any peak eluted at the retention time of drug.

\section{Precision:}

Precision is a major of the degree of repeatability (intraday), intermediate precision and reproducibility (Interday) of the analytical method under normal operating circumstances.

Precision is usually measured as the coefficient of variation or relative standard deviation of analytical results acquired from independently prepared samples (six tablets in case of dissolution).

Method precision was evaluated by performing the dissolution using proposed method (dissolution parameters and chromatographic method) on six tablets of UDCA tablets and calculated \% release of UDCA in each sample. The \%RSD for set of six tablets was calculated.

The intermediate precision of the method was also evaluated using different analyst and a different instrument in the same laboratory by carrying out dissolution on six more tablets using proposed method and calculated \% release of UDCA in each sample. Calculated the \%RSD for 12 results. The acceptance criteria for \%RSD was not more than 5\% and the absolute difference between results by two analysts was not more than $5 \%$.

\section{Recovery (accuracy):}

Accuracy is the closeness of agreement between test results and the accepted reference value. The accuracy of the method was performed by recovery studies. 
In order to evaluate the accuracy of the proposed method, recovery test was performed by adding known amount of standard solution to the placebo formulation sample, followed by analysis using the proposed dissolution parameters and chromatographic method.

The recovery studies were done at three different levels at $50 \%, 100 \%$ and $150 \%$ with three determinations of working level concentration using standard spiking method in placebo.

All the above solutions were prepared in duplicate; the sample aliquots were withdrawn after 60 minutes and were analyzed using proposed chromatographic condition. The recovery at each level was calculated by using the theoretical value from exact weight taken for spiking. The $\%$ recovery was calculated with respect to amount added. The acceptance criteria for \% recovery were - the recovery at each level should be in the range of $95 \%$ to $105 \%$ and overall \%RSD of nine results should be less than $5 \%$.

\section{Linearity:}

The linearity of an analytical method is its ability to elicit test results that are directly or by means of welldefined mathematical transformations, proportional to the concentration of analytes in the samples within a given range.

The linearity plot was constructed for UDCA in the concentration range of $50 \%$ to $150 \%$ of the sample concentration $(390 \mu \mathrm{g} / \mathrm{ml})$. The primary stock solution of UDCA working standard was prepared. From the primary stock solution, appropriate dilutions were made to get concentration of 190,290, 390, 490 and 590 $\mu \mathrm{g} / \mathrm{ml}$. The calibration curve was plotted as concentration of the respective drug solutions verses the peak area at each level. The results were statistically evaluated and correlation coefficient determination, slope and y-intercept values were calculated.

\section{Robustness:}

Robustness is a capacity of a method to remain unaffected by small deliberate changes in method parameters.

For robustness study, the standard concentration 550 $\mu \mathrm{g} / \mathrm{ml}$ of UDCA was used. Following chromatographic parameters were considered for the robustness study.

$>$ Effect of acetonitrile in mobile phase composition $( \pm 2 \%)$

$>$ Effect of flow rate $( \pm 0.1 \mathrm{ml} / \mathrm{min})$

$>$ Effect of wavelength $( \pm 2 \mathrm{~nm})$
The robustness of dissolution method was also evaluated by making deliberate changes in the dissolution parameters and analysis was performed using the proposed chromatographic method. The below dissolution parameters were changed during this study;

$>$ Change in $\mathrm{pH}$ of dissolution medium \pm 0.1

$>$ Change in RPM \pm 3 RPM

\section{Solution stability of sample and standard in dissolution medium:}

The solution stability of sample and standard solution in dissolution medium was performed to understand the stability which will be helpful for sample handling during routine analysis in laboratory by using the proposed method.

Solution stability was carried out for sample solution at 2-8 and ambient temperature for $24 \mathrm{hr}$. The sample and standard solution were injected immediately after preparation in the HPLC system considering it as an initial ( $0 \mathrm{hr}$.) as baseline.

\section{RESULTS AND DISCUSSION}

\section{Method Development}

Literature survey revealed that UDCA is having molecular weight $392.58 \mathrm{~g} / \mathrm{mol}$. The UDCA solution of $400 \mu \mathrm{g} / \mathrm{ml}$ concentration prepared in dissolution medium was scanned on PDA and the spectra of UDCA was recorded. It was observed that UDCA doesn't have good absorption after $210 \mathrm{~nm}$. Hence to detect the drug adequately the wavelength of $210 \mathrm{~nm}$ was selected as detection wavelength.

The blank (dissolution medium), placebo solution and sample solution was injected in HPLC system by using stationary phase $\mathrm{C} 18$ to check the interference due to diluent or placebo in main drug. The column of make

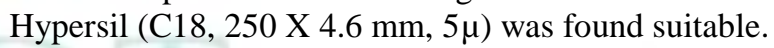

To achieve the optimum run time and capacity factor different compositions of organic modifier with buffer were tried at different $\mathrm{pH}$ buffer. The elution pattern of the UDCA was studied.

\section{Method Validation}

\section{Specificity:}

The chromatograms during specificity study (fig. 2, 3 and 4) of diluent, placebo and sample solution shows that there is no co elution of any peak with UDCA due to blank and placebo.

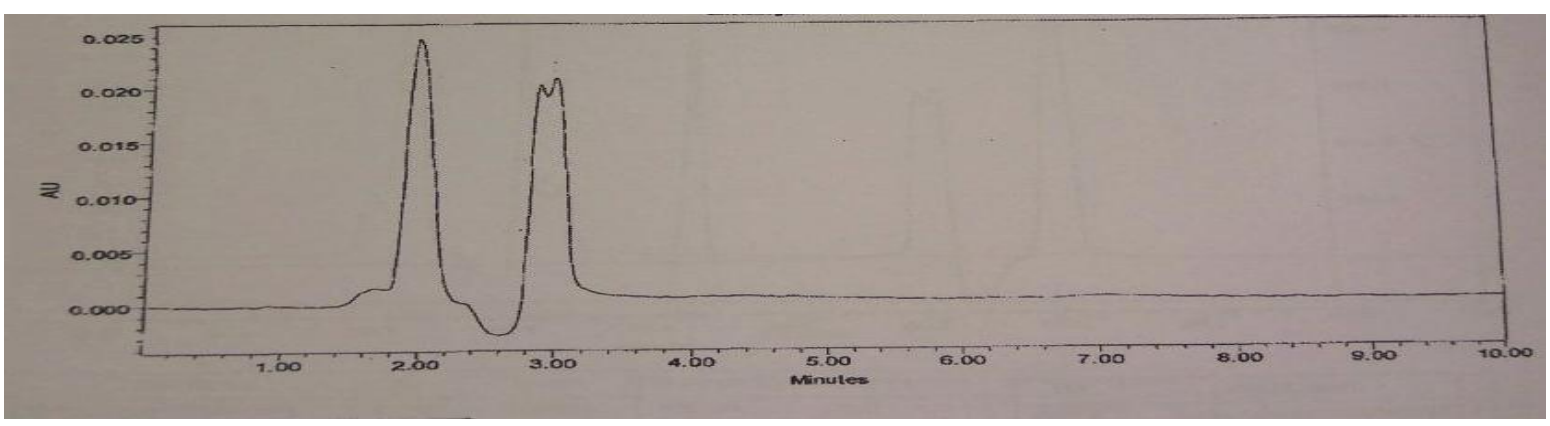

Figure 2: Blank Chromatogram 


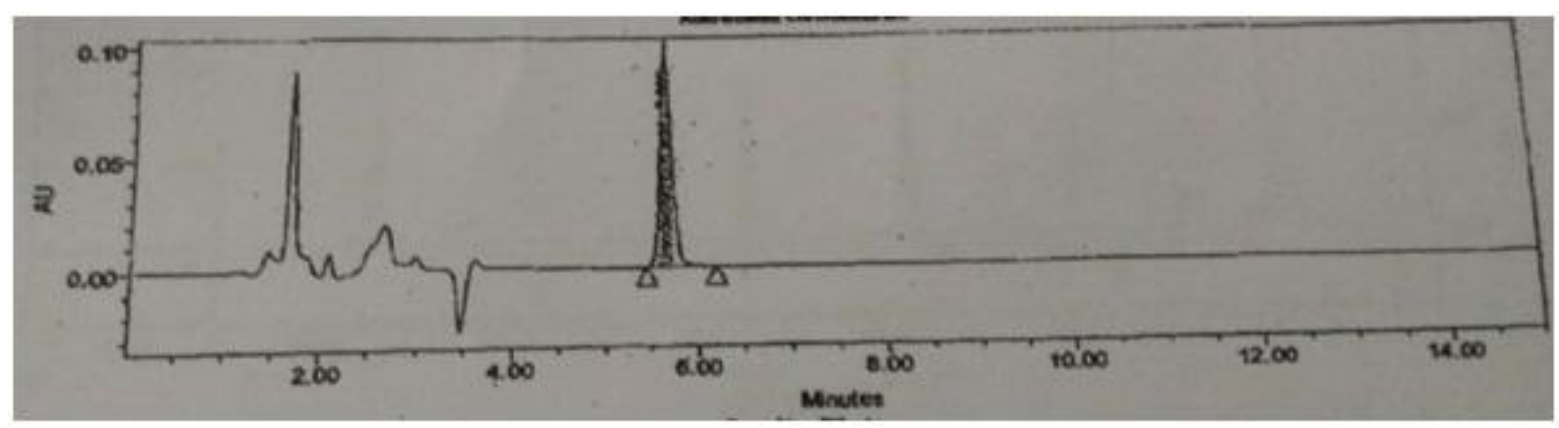

Figure 3: Standard Chromatogram

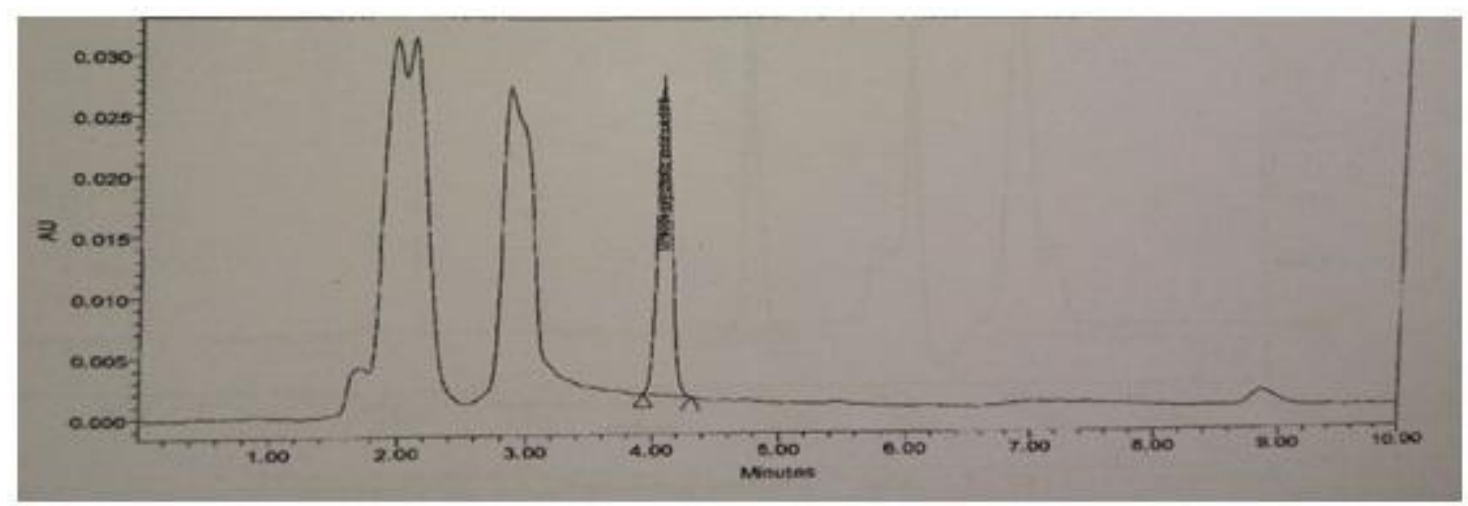

Figure 4: Standard Chromatogram

\section{Precision:}

\section{System Precision:}

The system precision was evaluated by injecting six standard solutions in HPLC by using the proposed chromatographic conditions and the \% RSD of Area and retention time was calculated. The $\%$ RSD of area was found $0.85 \%$, and $\%$ RSD of retention time was found $0.43 \%$.

\section{Method Precision:}

Method precision was divided in two parts as below;

Intraday precision: Intraday precision was evaluated by performing dissolution of Ursodeoxycholic acid $300 \mathrm{mg}$ tablets on six tablets $(n=6)$ using the proposed dissolution and chromatographic parameters, \% drug release was calculated. The \%RSD for six results (of six tablets) was calculated and found $1.6 \%$.

\section{Inter-day precision (Ruggedness):}

The ruggedness of the method was evaluated by performing dissolution of Ursodeoxycholic acid $300 \mathrm{mg}$ tablets on six tablets (n=6) using the proposed dissolution and chromatographic parameters on different day and on different instrument. The $\%$ drug release was calculated and \%RSD of six results was found $1.5 \%$. The inter-day precision for two sets $(n=12)$ for their $\%$ recovery was found to be $1.5 \%$. The absolute difference between results for intermediate precision was found $2 \%$ (table 1 ).

Table 1: Method Precision results

\begin{tabular}{|l|l|l|}
\hline Sample No. & \% Dissolution (Precision) & \% Dissolution (Intermediate Precision) \\
\hline 1 & 98 & 95 \\
\hline 2 & 96 & 96 \\
\hline 3 & 99 & 99 \\
\hline 4 & 98 & 95 \\
\hline 5 & 100 & 96 \\
\hline 6 & 96 & 96 \\
\hline Mean & 98 & 96 \\
\hline \%RSD & 1.6 & 1.5 \\
\hline Overall mean & 97 \\
\hline Over all \%RSD & 1.5 \\
\hline Absolute difference of assay results & $2 \%$ \\
\hline
\end{tabular}




\section{Accuracy (Recovery):}

The $\%$ recovery at $50 \%, 100 \%$ and $150 \%$ was found to be $99.4 \pm 0.38,99.6 \pm 0.36$ and $99.8 \pm 0.35 \%$ respectively (table 3 ). The overall mean recovery was found to be
$99.6 \pm 0.38 \%$. The recovery results were found within acceptance criteria (table 3 ). The developed method found to be accurate for determination of drug release of UDCA in dissolution samples of Ursodeoxycholic Acid tablets.

Table 2: Accuracy

\begin{tabular}{|l|l|l|l|l|}
\hline Level & Sample no. & \% Recovery & Mean & \%RSD \\
\hline \multirow{4}{*}{$50 \%$} & 1 & 99.2 & 99.4 & \\
\cline { 2 - 4 } & 2 & 99.8 & & \\
\cline { 2 - 3 } & 3 & 99.1 & & \\
\hline \multirow{3}{*}{$100 \%$} & 1 & 99.5 & & \\
\cline { 2 - 3 } & 2 & 100.0 & & \multirow{2}{*}{0.36} \\
\cline { 2 - 3 } & 3 & 99.3 & & \\
\hline \multirow{3}{*}{$150 \%$} & 1 & 99.5 & & \\
\cline { 2 - 3 } & 2 & 99.8 & & \\
\cline { 2 - 3 } & 3 & 100.2 & & \\
\hline
\end{tabular}

\section{Linearity:}

Linearity was evaluated at five different (50\% to $150 \%$ ) level considering the working concentration level as $100 \%$. The area response of UDCA drug against concentration at five different level was performed in the range of $50 \%-150 \%$ and response for UDCA found to be linear. The correlation coefficient $(r)$ was found to be 0.999 . The linearity was found with in acceptance criteria.

Robustness:
The robustness of the method was verified by making the deliberate changes in the critical chromatographic parameters and the system suitability was checked. Below chromatographic parameters (one parameter at a time) were changed.

$>$ Organic modifier composition

$>$ Pump flow rate

$>$ Column oven temperature

The results are presented in table -3 and found within the acceptance criteria.

Table 3: Robustness Results for chromatographic parameter

\begin{tabular}{|l|c|c|}
\hline \multicolumn{2}{|c|}{ HPLC Parameter } & \% RSD of standard \\
\hline Initial & As per actual method & 0.62 \\
\hline $\begin{array}{l}\text { Acetonitrile composition in mobile } \\
\text { phase }( \pm 2 \%)\end{array}$ & $+2 \%(52 \%)$ & 0.45 \\
\cline { 2 - 3 } Pump flow rate $\pm 0.1 \mathrm{ml} / \mathrm{min}$ & $-2 \%(48 \%)$ & 0.52 \\
\cline { 2 - 3 } & $1.1 \mathrm{ml} / \mathrm{min}$ & 0.44 \\
\hline Wavelength $\pm 2 \mathrm{~nm}$ & $0.9 \mathrm{ml} / \mathrm{min}$ & 0.21 \\
\cline { 2 - 3 } & $208 \mathrm{~nm}$ & 0.15 \\
\hline
\end{tabular}

The robustness of the dissolution method was evaluated by making the changes in proposed dissolution parameters.
The results are presented in table -4 and found within the acceptance criteria.

Table 4: Robustness results for dissolution parameter

\begin{tabular}{|c|c|c|c|}
\hline \multicolumn{2}{|c|}{ Dissolution Parameter } & \% Average Drug Release & $\%$ RSD of six tablets \\
\hline Initial & As per actual method & 95 & 2.8 \\
\hline \multirow{2}{*}{$\begin{array}{l}\mathrm{pH} \text { of Dissolution Medium } \\
( \pm 0.1)\end{array}$} & $+0.1(7.6)$ & 97 & 3.2 \\
\hline & $-0.1(7.4)$ & 92 & 2.9 \\
\hline \multirow{2}{*}{$\begin{array}{l}\text { Rotation of paddle per minute } \\
\pm 0.1 \mathrm{ml} / \mathrm{min}\end{array}$} & +3 (78 RPM) & 93 & 1.8 \\
\hline & -3 (72 RPM) & 95 & 3.0 \\
\hline
\end{tabular}

\section{Solution Stability of Sample and Standard}

The Ursodeoxycholic Acid sample found to be stable up to 24 hours in dissolution medium at ambient temperature. The average $\%$ results after 24 hours were found to be $95 \%$. 


\section{Application of developed method:}

The proposed dissolution method with chromatographic method was used for measurement of $\%$ drug release of Ursodeoxycholic Acid tablets 500mg, the results were found within the specification. The proposed method can be used in laboratory for dissolution of Ursodeoxycholic Acid tablets.

\section{CONCLUSION}

The proposed HPLC method is accurate, linear, precise and rugged methodology for quantitative determination (Assay) of Ursodeoxycholic Acid in Ursodeoxycholic Acid tablets of different strengths. The results of the

\section{REFERENCES}

1. Bouchier IAD: The medical treatment of gallstones. Annu Res Med 1980; 31:59-77.

2. Dowling RH: Cholelithiasis: medical treatment. Clin Gastroenterol 1983; 12:125-178.

3. Bergmann K, Epple-Gutsfeld M, Leiss O: Differences in the effects of chenodeoxycholic acid and ursodeoxycholic acid on biliary lipid secretion and bile acid synthesis in patients with gallstones. Gastroenterology: 1984; 87: 136-143.

4. Leuschner U, Leuschner M, Hubner K: Gallstone dissolution with patients with chronic active hepatitis. Gastroenterology: 1981; 80: 1208-1209.

5. Mukharjee J, Pal TK, Development and Validation of RP_HPLC method to determine Ursodeoxycholic Acid in Pharmaceutical Dosage Forms; IJPSR, 20111; 2(1):73-78.

6. Peepliwal A, Bonde CG, Bothara KG. "A validated RP-HPLC method for quantitative determination of related impurities of ursodeoxycholic acid (API) by refractive index detection." Journal of pharmaceutical and biomedical analysis 2011; 54(4): 845-849. method validation study show that the method is accurate and linear in the concentration range of 50\%$150 \%$ of Ursodeoxycholic Acid. The analytical method validation was done as per ICH Q2(R1) and USP guidelines. This method can be used by quality control or research laboratories for quantitative determination of the content (assay) of Ursodeoxycholic Acid tablets of different strength.

\section{ACKNOWLEDGEMENTS}

The authors thank the Principal, Shri Chhatrapati Shivaji College, Omerga, Dr. Babasaheb Ambedkar, Marathwada University, India for providing support and facilities during research work.

7. Shen, Zhaojun, et al. "A Validated RP-HPLC Method for Quantitative Determination of Related Impurities of Cholic Acid Bulk Drugs." Acta Chromatographica 2017; 1-5.

8. Won Park Je, Nguyen Huu Hoang, and Nguyen Lan Huong. "Analysis of Ursodeoxycholic Acid Using Ultra-Performance Liquid Chromatography with Tandem Mass Spectrometry." Current Pharmaceutical Analysis 2016; 12(3):185-191.

9. Hasan N, et al. "Development and Validation of RP-LC-UV Method for Determination of Ursodeoxycholic Acid in Capsule and Human Serum." World Applied Sciences Journal, 2014; 32(4):560-566.

10. International Conference on Harmonization Harmonized Tripartite Guideline. (2005) Validation of Analytical procedures: Q2(R1).

11. United States of Pharmacopoeia 38 NF 33 (2015). United States Pharmacopeial Convention General chapter <1225>, Validation of Compendial Procedures, pp. 1445-1450.

12. United States of Pharmacopoeia 38 NF 33 (2015), United States Pharmacopeial Convention General chapter <621>, Chromatography, pp. 424-434. 